\title{
POINTWISE ESTIMATES FOR THE RELATIVE FUNDAMENTAL SOLUTION OF $\bar{\partial}_{b}$
}

\author{
MICHAEL CHRIST
}

(Communicated by Walter Littman)

\begin{abstract}
Consider a compact pseudoconvex $C R$ manifold of dimension 3 and finite type, on which the operator $\bar{\partial}_{b}$ has closed range in $L^{2}$. The relative fundamental solution of $\bar{\partial}_{b}$ is the distribution-kernel for that operator which inverts $\bar{\partial}_{b}$, modulo its kernel and cokernel. We derive pointwise bounds on this fundamental solution and its derivatives.
\end{abstract}

Let $M$ be a compact $C R$ manifold of real dimension 3. We assume that $M$ is pseudoconvex and of finite type $m$, and that the $\bar{\partial}_{b}$ operator on $M$ has closed range on $L^{2}$. The latter holds automatically when $M$ is the boundary of a smooth, bounded pseudoconvex domain in $C^{2}$. Fix a positive measure on $M$ with a smooth, nonvanishing density in local coordinates. Let $S$ denote the Szegö projection of $L^{2}$, with respect to this measure, onto the kernel $H_{b}$ of $\bar{\partial}_{b}$ in $L^{2}$. $\bar{\partial}_{b}$ maps (test) functions to sections of a bundle $B^{0,1}$; fix an inner product structure on the bundle and let $L^{2 *}$ denote the Hilbert space of $L^{2}$ sections of $B^{0,1}$. Let $\bar{\partial}_{b^{*}}$ denote the adjoint operator, let $S^{*}$ denote the orthogonal projection of $L^{2 *}$ onto the kernel $H_{b^{*}} \subset L^{2 *}$ of $\bar{\partial}_{b^{*}}$, and let $K, K^{*}$ be the distribution-kernels for $S, S^{*}$ respectively. For definitions of all these terms and references see for instance $[\mathbf{C}],[\mathbf{F K}],[\mathbf{K}]$.

The hypothesis that $\bar{\partial}_{b}$ has closed range means that Range $\left(\bar{\partial}_{b}\right)=L^{2 *} \cap \bar{\partial}_{b}\left(L^{2}\right)$ is a closed subspace of $L^{2 *}$, and that for each $f \in \operatorname{Range}\left(\bar{\partial}_{b}\right)$ there exists a unique $u \in L^{2}$ satisfying

$$
\left\{\begin{array}{l}
\bar{\partial}_{b} u=f \\
u_{b} \perp H_{b}
\end{array}\right.
$$

Moreover $\|u\|_{2} \leq C\|f\|_{2}$. Therefore the operator $G$ which maps any $f \in L^{2 *}$ to the unique $u \perp H_{b}$ satisfying $\bar{\partial}_{b} u=\left(I-S^{*}\right) f$, is bounded from $L^{2 *}$ to $L^{2}$. Let $L$ denote its distribution-kernel, the relative fundamental solution for $\bar{\partial}_{b}$. The purpose of this article is to obtain certain pointwise bounds for $L$ and its derivatives. This is a continuation of the work $[\mathbf{C}]$ and is based on the results obtained there; we shall continue to employ the notation of that paper without full explanation. In particular the bounds we seek for $L$ are formulated in terms of a quasi-metric $\rho$ and a family of balls $B(x, r)$ on $M$, constructed and studied in the fundamental paper [NSW], which are induced by the $C R$ structure in a natural way. In this connection $\hat{B}$ denotes the unit ball in $\mathbf{R}^{3}$, and for each $x \in M, r \in\left(0, C_{M}\right]$ there is given a special coordinate map $\phi_{x, r}$, a diffeomorphism of $\hat{B}$ onto $B(x, r) . \Lambda(x, r)$

Received by the editors December 1, 1987.

1980 Mathematics Subject Classification (1985 Revision). Primary 35A08, 35N15; Secondary $32 \mathrm{~F} 30$.

This research was supported by the Mathematical Sciences Research Institute. 
denotes the measure of $B(x, r)$. For a summary of their relevant properties see section 15 of $[\mathbf{C}]$.

In local coordinates in $M, \bar{\partial}_{b}$ takes the form $X+i Y$ where $X, Y$ are real, smooth vector fields, linearly independent at every point. Define, for $x, y$ in a coordinate patch, $\vartheta(x, y)$ to be the infimum of all $r$ such that there exists an absolutely continuous function $\psi$ from $[0, r]$ into the coordinate patch, with $\psi(0)=x$ and $\psi(r)=y$, such that for almost all $t, d \psi / d t=a(t) X(\psi(t))+b(t) Y(\psi(t))$, with $a^{2}(t)+b^{2}(t) \leq 1$. Then $\vartheta(x, y)$ is finite, and there is a uniform inequality $C \rho(x, y) \leq \vartheta(x, y) \leq C^{\prime} \rho(x, y) . \quad B(x, r)$ is $\{y: \rho(x, y)<r\}$. Equivalent reformulations of the estimates below may be obtained by replacing $\rho$ by $\vartheta$ and $B(x, r)$ by $\{y: \vartheta(x, y)<r\}$; the measures of $B(x, r)$ and $\{y: \vartheta(x, y)<r\}$ are comparable, uniformly in $x$ and $r$.

We denote by $D$ any differential operator of the form $(X$ or $Y) \circ(X$ or $Y) \ldots$ and let $n$ be the number of factors of $(X$ or $Y)$, possibly zero. $D_{x}$ denotes such an operator acting in the $x$-variable, with $n$ factors, and $D_{y}$ acts in the $y$-variable and has $n^{\prime}$ factors.

Our main result is

THEOREM 1. L is $C^{\infty}$ away from the diagonal

$$
\left|D_{x} D_{y} L(x, y)\right| \leq C_{n, n^{\prime}} r^{1-n-n^{\prime}} \Lambda(x, r)^{-1}
$$

uniformly for all $n, n^{\prime}$ and $x \neq y \in M$, where $r=\rho(x, y)$.

An immediate consequence is

THEOREM 2. Suppose that $f \in L^{2} *, u \perp H_{b}$ and $\bar{\partial}_{b} u=f$. Suppose further that $f$ is bounded on some open set $U \subset M$. Then $u$ is Hölder continuous of order $m^{-1}$ on every compact subset of $U$.

This follows directly from the first theorem, by Theorem 14(b) of [RS]. Moreover Theorem 1 implies that $|u(x)-u(y)| \leq C \rho(x, y) \log \left(\rho(x, y)^{-1}\right)$ as $\rho(x, y)$ tends to 0 . (Recall that $\rho(x, y) \leq C|x-y|^{\delta}$, where $\delta=m^{-1}$ [NSW].) Under the hypothesis of type $m$, this is the best order of regularity that could be concluded, even if it were known that $X u, Y u$ were separately bounded on $U$. Thus the results of Theorem 1 are fairly sharp. Theorem 2 has also been obtained by Fefferman and Kohn [FK].

To begin the proofs observe that $L$ is $C^{\infty}$ away from the diagonal. For the distribution-kernel for $I-S^{*}$ is $C^{\infty}$ off of the diagonal (see below), and the solution $u \perp H_{b}$ of $\bar{\partial}_{b} u=h$ is $C^{\infty}$ wherever $h$ is. See [K] (or [C]). It remains to examine $L$ near the diagonal.

Consider any distinct points $x_{0}, y_{0}$ in a common coordinate patch, close together. let $c_{1} \ll 1 \ll c_{2}$ be two constants depending only on $M$, very sinall and very large respectively. Let $r=\rho\left(x_{0}, y_{0}\right), B=B\left(x_{0}, c_{2} r\right), B_{1}=B\left(y_{0}, c_{1} r\right), B_{3}=B\left(y_{0}, 2 c_{1} r\right)$, $B_{2}=B\left(x_{0}, c_{1} r\right)$, and $B_{4}=B\left(x_{0}, 2 c_{1} r\right)$. To analyze $L$ and its $x$-derivatives at $\left(x_{0}, y_{0}\right)$ we consider the map from $L^{2 *}\left(B_{1}\right)$ to $C^{\infty}\left(B_{2}\right)$ which sends any $f \in L^{2 *}$ supported on $B_{1}$ to $G f$ restricted to $B_{2}$. Let $u=G f$.

The first step is to analyze $\left(I-S^{*}\right) f$. In $[\mathbf{C}]$ was proved

THEOREM A. $K^{*}$ is $C^{\infty}$ away from the diagonal and satisfies

$$
\left|D_{x} D_{y} K^{*}(x, y)\right| \leq C_{n, n^{\prime}} \rho(x, y)^{-n-n^{\prime}} \Lambda(x, \rho(x, y))^{-1}
$$

for all $D_{x}, D_{y}$. The same holds for $K$. 
Let $h=\left(I-S^{*}\right) f$ and $\hat{h}=h \circ \phi_{x_{0}, c_{2} r}$ on $\hat{B}$. Let $\hat{X}$ and $\hat{Y}$ be the pullbacks of $X, Y$ and let $\hat{D}=(\hat{X}$ or $\hat{Y}) \circ(\hat{X}$ or $\hat{Y}) \ldots$ with $n$ factors on $\hat{B}$. From Theorem $\mathrm{A}$ and the restriction that $f$ be supported on $B_{1}$ there easily follows

$$
\|D h\|_{L^{2}\left(B_{2}\right)} \leq C_{n} r^{-n}\|f\|_{2} .
$$

Equivalently

COROLlaRY 3 .

for all $n \geq 0$.

$$
\|\hat{D} \hat{h}\|_{L^{2}\left(\hat{B}_{2}\right)} \leq C_{n} \Lambda\left(x_{0}, r\right)^{-1 / 2}\|f\|_{2}
$$

For each $x_{0}, y_{0}$ there exists $\hat{\psi} \in C_{0}^{\infty}(\hat{B})$ with $C^{k}$ norm bounded uniformly in $x_{0}, y_{0}$ for all $k$, satisfying $\hat{\psi} \equiv 1$ on $\phi^{-1}\left(B_{4}\right)$ and $\hat{\psi} \equiv 0$ on $\phi^{-1}\left(B_{3}\right)$, where $\phi=$ $\phi_{x_{0}, c_{2} r}$. Let $\psi=\hat{\psi} \circ \phi^{-1}$, which may be viewed as a $C^{\infty}$ function on $M$ supported on $B_{4}$ by virtue of the compact support of $\hat{\psi}$. Let $v \in L^{2 *}$ be the unique solution of

$$
\left\{\begin{array}{l}
\bar{\partial}_{b^{*}} v=(I-S)(\psi u) \\
v \perp H_{b^{*}}
\end{array}\right.
$$

$I-S$ projects onto Range $\left(\bar{\partial}_{b^{*}}\right)$, the orthocomplement of $H_{b}$, so a solution exists.

LEMMA 4.

$$
\|\psi u\|_{2} \leq C r\|f\|_{2}
$$

and

uniformly for all $x_{0}, y_{0}, f$.

$$
\|v\|_{L^{2}(B)} \leq C r^{2}\|f\|_{2}
$$

This is an immediate consequence of

Proposition B [C]. If $f \in \operatorname{Range}\left(\bar{\partial}_{b}\right)$ and $u \perp H_{b}$ * satisfies $\bar{\partial}_{b} u=f$ then

$$
\|u\|_{L^{2}(B(x, r))} \leq C r\|f\|_{2}
$$

uniformly for all $x \in M, r>0, f$. The corresponding estimate is valid for the $\bar{\partial}_{b^{*}}$. equation.

$v$ is introduced in order to obtain the factor of $r^{2}$ in Lemma 4, which permits the rescaling argument below. It would be more natural to consider the solution $w$ of $\bar{\partial}_{b^{*}} w=u$ with $w \perp H_{b^{*}}$, but we do not know that $\|w\|_{L^{2}(B)} \leq C r^{2}\|f\|_{2}$. Otherwise Theorem 1 would be an immediate consequence of the arguments in $[\mathbf{C}]$.

Restrict everything to $B_{4}$, and let $z=(I-S)(\psi u)$. Then

$$
\left\{\begin{array}{l}
\bar{\partial}_{b^{*}} v=z, \\
\bar{\partial}_{b} z=h
\end{array}\right.
$$

with the $L^{2}\left(B_{4}\right)$ norms satisfying, for all $n$,

$$
\left\|r^{-2} v\right\|_{2}+\left\|r^{-1} z\right\|_{2}+\left\|r^{n} D h\right\|_{2} \leq C_{n}\|f\|_{L^{2}(M)} .
$$

Now pull everything back to $\hat{B}$ via $\phi=\phi_{x_{0}, 2 c_{1} r}$. Let $\hat{g}=g \circ \phi$ for any $g$ defined on $B$, and let $\hat{\partial}$ and $\hat{\partial}_{*}$ be the pullbacks of $\bar{\partial}_{b}$ and $\bar{\partial}_{b^{*}}$, respectively. Then the equations rescale to

$$
\left\{\begin{array}{l}
\hat{\partial}_{*}\left(r^{-2} \hat{v}\right)=\left(r^{-1} \hat{z}\right) \\
\hat{\partial}\left(r^{-1} \hat{z}\right)=\hat{h}
\end{array}\right.
$$


with the control

$$
\left\|r^{-2} \hat{v}\right\|_{2}+\left\|r^{-1} \hat{z}\right\|_{2}+\|\hat{D} \hat{h}\|_{2} \leq C_{n} \Lambda\left(x_{0}, r\right)^{-1 / 2}\|f\|_{2}
$$

for all $n$, uniformly in $x_{0}, y_{0}$.

It is proved in $[\mathbf{K}]$ (see also $[\mathbf{C}]$ ) that this implies

$$
\left\|r^{-1} \hat{z}\right\|_{C^{k}} \leq C_{k} \Lambda\left(x_{0}, r\right)^{-1 / 2}\|f\|_{2}
$$

on any fixed compact subset of $\hat{B}$, for all $k$. Therefore on the inverse image of $B_{2}$

$$
\left\|\hat{D} r^{-1} \hat{z}\right\|_{L^{\infty}(\hat{B})} \leq C_{n} \Lambda\left(x_{0}, r\right)^{-1 / 2}\|f\|_{2}
$$

which is to say that

$$
\|D[(I-S)(\psi u)]\|_{L^{\infty}\left(B_{2}\right)} \leq C_{n} r^{1-n} \Lambda\left(x_{0}, r\right)^{-1 / 2}\|f\|_{2}
$$

for all $D$.

$D[u-(I-S)(\psi u)]$ may be estimated more directly, on $B_{2}$. Let $u_{j}$ be the restriction of $u$ to $B\left(x_{0}, 2^{j} r\right) \backslash \bigcup_{i<j} B\left(x_{0}, 2^{i} r\right) . \quad u=(I-S) u$ since $u \perp H_{b}$, so $[u-(I-S)(\psi u)]=(I-S)[(1-\psi) u]$. Hence on $B_{2}$

$$
[u-(I-S)(\psi u)]=-\sum_{j=0}^{\infty} S u_{j}
$$

Fix any $D_{x}$ and let $K_{j}$ be the restriction of $D_{x} K$ to $\left\{(x, y): x \in B_{2}\right.$ and $\rho(x, y) \sim$ $2^{j} r$ so that $D_{x} S u_{j}=\int K_{j}(x, y) u_{j}(y) d y$ on $B_{2}$. Then

$$
\begin{aligned}
\left\|D_{x} S u_{j}\right\|_{L^{\infty}\left(B_{2}\right)} & \leq C \sup _{x}\left\|K_{j}(x, \cdot)\right\|_{\infty}\left\|u_{j}\right\|_{1} \\
& \leq C\left(2^{j} r\right)^{-n} \Lambda\left(x_{0}, 2^{j} r\right)^{-1}\left\|u_{j}\right\|_{2} \Lambda\left(x_{0}, 2^{j} r\right)^{1 / 2} \\
& \leq C\left(2^{j} r\right)^{1-n} \Lambda\left(x_{0}, 2^{j} r\right)^{-1 / 2}\|f\|_{2} .
\end{aligned}
$$

We have used Theorem A to estimate $K_{j}$ and Proposition B to estimate $\left\|u_{j}\right\|_{2}$, and have used the facts that $\rho$ satisfies a quasi-triangle inequality, and that $\Lambda\left(x, C 2^{j}\right) \approx$ $\Lambda\left(x_{0}, 2^{j}\right)$ for $x \in B_{2} . \Lambda\left(x_{0}, 2^{j} r\right) \geq C 2^{4 j} \Lambda\left(x_{0}, r\right)[C, \S 15]$, so

$$
\begin{aligned}
\left\|D_{x}[u-(I-S)(\psi u)]\right\|_{L^{\infty}\left(B_{2}\right)} & \leq C r^{1-n} \Lambda\left(x_{0}, r\right)^{-1 / 2}\|f\|_{2} \cdot \sum_{j \geq 0} 2^{j(1-n)} 2^{-2 j} \\
& \leq C r^{1-n} \Lambda\left(x_{0}, r\right)^{-1 / 2}\|f\|_{2} .
\end{aligned}
$$

Together with (3), since $u(x)=\int L(x, y) f(y) d y$, this establishes

LEMMA 5. For all distinct $x_{0}, y_{0} \in M$ and all $D_{x}$

$$
\left\|D_{x} L(x, \cdot)\right\|_{L^{2}\left(B\left(y_{0}, c_{1} r\right)\right)} \leq C_{n} r^{1-n} \Lambda\left(x_{0}, r\right)^{-1 / 2}
$$

for all $x \in B\left(x_{0}, c_{1} r\right)$ where $r=\rho\left(x_{0}, y_{0}\right)$. 
The next claim is that the same holds with the roles of the variables reversed:

$$
\left\|D_{y} L(\cdot, y)\right\|_{L^{2}\left(B\left(x_{0}, c_{1} r\right)\right)} \leq C r^{1-n^{\prime}} \Lambda\left(x_{0}, r\right)^{-1 / 2}
$$

for all $y \in B\left(y_{0}, c_{1} r\right)$. $\left(\Lambda\left(x_{0}, r\right) \sim \Lambda\left(y_{0}, r\right)\right.$ so the lack of symmetry is only apparent.) Observe that the adjoint $G^{*}$ of $G$ is the operator which first maps any $g \in L^{2}$ to $(I-S) g$, then sends it to the solution $v \perp H_{b^{*}}$ of $\bar{\partial}_{b^{*}} v=(I-S) g$; in other words the distribution-kernel for $G^{*}$ is the relative fundamental solution for $\bar{\partial}_{b^{*}}$. Since the whole machine applies equally well to $\bar{\partial}_{b^{*}}$ as to $\bar{\partial}_{b},(4)$ follows from a repetition of the proof of Lemma 5. To verify the observation note that $G^{*}=\left(I-S^{*}\right) G^{*}(I-S)$ since $G=(I-S) G\left(I-S^{*}\right)$. Thus it suffices to show that $\bar{\partial}_{b^{*}} \circ G^{*}$ is the identity on the orthocomplement of $H_{b^{*}}$. But $G \bar{\partial}_{b}=(I-S)$ on test functions by definition, so $\bar{\partial}_{b^{*}} \circ G^{*}=(I-S)$.

Finally pull $L$ back to $\hat{L}$ on $\hat{B} \times \hat{B}$ via $\phi_{x_{0}, c_{1} r} \times \phi_{y_{0}, c_{1} r}$. Lemma 5 and (4) pull back to

$$
\sup _{\xi}\left\|\hat{D}_{\xi} \hat{L}(\xi, \cdot)\right\|_{2} \leq C_{n} r \Lambda\left(x_{0}, r\right)^{-1}
$$

and

$$
\sup _{\eta}\left\|\hat{D}_{\eta} \hat{L}(\cdot, \eta)\right\|_{2} \leq C_{n^{\prime}} r \Lambda\left(x_{0}, r\right)^{-1}
$$

for all $\hat{D}_{\xi}, \hat{D}_{\eta}$, where the suprema are taken over $\hat{B}$. The additional factor of $\Lambda\left(x_{0}, r\right)^{-1 / 2}$ comes from the change of variables. Thus

$$
\|\left(\hat{D}_{\xi} \text { or } \hat{D}_{\eta}\right) \hat{L} \|_{2} \leq C r \Lambda\left(x_{0}, r\right)^{-1 / 2}
$$

with a bound which depends only on $M$ and on the orders of $\hat{D}_{\xi}$ and $\hat{D}_{\eta} . \hat{X}$ and $\hat{Y}$ together with all their commutators of length at most $m$ span the tangent space at each point of $\hat{B}$, and do so in a uniform way. Therefore by standard elliptic theory

$$
\|\hat{L}\|_{C^{n}} \leq C_{n} r \Lambda\left(x_{0}, r\right)^{-1}
$$

on any fixed compact subset of $\hat{B} \times \hat{B}$. Passing back to $M \times M$ gives

$$
\left|D_{x} D_{y} \hat{L}\left(x_{0}, y_{0}\right)\right| \leq C_{n, n^{\prime}} r^{1-n-n^{\prime}} \Lambda\left(x_{0}, r\right)^{-1}
$$

for all $D_{x}, D_{y}$ as desired.

Observe that because of the relations $\bar{\partial}_{b} G=(I-S)$ and $\bar{\partial}_{b^{*}} G^{*}=\left(I-S^{*}\right)$, Theorem 1 implies Theorem A. It also implies Proposition B.

I am grateful to D. Jerison and E. M. Stein for pointing out an error in the original manuscript.

\section{REFERENCES}

[C] M. Christ, Regularity properties of the $\partial_{b}$ equation on weakly pseudoconvex $C R$ manifolds of dimension 3, J. Amer. Math. Soc. (to appear).

[FK] C. Fefferman and J. J. Kohn, Hölder estimates on domains of complex dimension two and on three dimensional CR manifolds, Adv. in Math. 69 (1988), pp. 223-303.

[K] J. J. Kohn, Estimates for $\partial_{b}$ on pseudoconvex CR manifolds, Proc. Sympos. Pure Math., vol. 43, Amer. Math. Soc., Providence, R.I., 1985, pp. 207-217.

[M] M. Machedon, Szegö kernels on pseudoconvex domains with one degenerate eigenvalue, preprint.

[NSW] A. Nagel, E. M. Stein and S. Wainger, Balls and metrics associated to vector fields, I: Basic properties, Acta Math. 155 (1985), 103-147. 
[RS] L. P. Rothschild and E. M. Stein, Hypoelliptic differential operators and nilpotent groups, Acta Math. 137 (1976), 247-320.

[S] A. Sánchez-Calle, Fundamental solutions and geometry of the sum of squares of vector fields, Invent. Math. 78 (1984), 143-160.

Department of Mathematics, University of California, Los Angeles, CaliFORNIA 90024 